\title{
Effect of low-dose aspirin on candidate biomarkers in the urine proteome
}

\begin{abstract}
A biomarker is a measurable change associated with a physiological or pathophysiological process. Unlike blood, which is homeostatic, urine is more likely to reflect changes in the body; urine is also a promising source of biomarker discovery because changes in physiology are accumulated in this fluid. However, urinary biomarker discovery remains a major challenge because the urinary proteome is influenced by various factors. Low-dose aspirin is widely used for preventing myocardial infarction, ischemic stroke and vascular death among patients at both high and low risk for these events as well as some cancers. Because animal models allow for controlling genetic and environmental factors, in this study, the effects of aspirin on the urinary proteome were analysed in rats. Urinary samples were collected before and at 7 days after administration of low-dose aspirin $(50 \mathrm{mg} / \mathrm{kg} . \mathrm{d})$. A total of 191 proteins were identified by Nano LC-MS/MS and then quantified using Scaffold software. Twelve of these proteins were significantly altered, suggesting that aspirin affects the urinary proteome. Nine of the 12 proteins have been previously reported as candidate disease biomarkers, suggesting that these effects must be considered in future urinary biomarker research. Additionally, the changes in protein induced by low-dose aspirin were distinct from those caused by heparin and argatroban treatments; this indicates that the urinary proteome can distinguish blood coagulation status induced by different coagulation mechanisms, which may help in the design of more specific treatment plans for patients. Two significantly up-regulated proteins, pro-EGF and CD10, were selected for further validation by western blotting, and the results were consistent with those of mass spectrometry. Therefore, we propose that these two proteins may be used for monitoring platelet aggregation.
\end{abstract}

Volume 3 Issue 3 - 2016

\author{
Xundou Li, 'Youhe Gao' \\ 'National Key Laboratory of Medical Molecular Biology, Chinese \\ Academy of Medical Sciences/Peking Union Medical College, \\ China \\ ${ }^{2}$ Department of Biochemistry and Molecular Biology, Beijing \\ Normal University, China
}

\author{
Correspondence: Youhe Gao, Department of Biochemistry \\ and Molecular Biology, Beijing Normal University, Gene \\ Engineering and Biotechnology Beijing Key Laboratory, Beijing, \\ 100875, PR. of China, Tel 86I058804382. \\ Email gaoyouhe@bnu.edu
}

Received: February 28, 2016 | Published: March 24, 2016

Keywords: low-dose aspirin, urinary proteome

Abbreviations: LC-MS/MS, liquid chromatography-tandem mass; 1D-SDS PAGE, one dimensional sodium dodecyl sulfate polyacrylamide gel electrophoresis; DTT, dithiothreitol; CAN, acetonitrile; IAA, iodoacetamide

\section{Introduction}

A biomarker is a measurable change associated with a physiological or pathophysiological process. Unlike blood, which is homeostatic, urine is more likely to reflect changes in the body. In addition, a variety of physiological changes can accumulate in urine, making it a good biological source for disease biomarker discovery. ${ }^{1,2}$ As various physiological changes can be detected in urine, we expect that urine can be used to detect small, early changes in pathological and/or pharmacological conditions. ${ }^{3}$ Indeed, urine can be more sensitive to physiological changes than blood, ${ }^{4}$ and several potential biomarkers perform even better in urine than in blood..$^{5-7}$ Many urinary biomarkers have recently been identified for various types of diseases. ${ }^{8}$ Because the urinary proteome can be affected by a variety of factors such as gender, ${ }^{9}$ age, medications, ${ }^{10,11}$ exercise, ${ }^{12}$ and smoking ${ }^{13}$ in addition to many other physiological variables and environmental factors, urinary biomarker discovery remains challenging. Some factors are easily eliminated or balanced by parallel-group trials and utilization of a large sample size in clinical urinary samples. Nonetheless, to facilitate the identification of disease-specific urinary biomarkers, it is essential to understand the effects of commonly used medications on the urinary proteome. To achieve this objective, it is important to employ simple and controllable systems, such as animal models. Because animal models allow for controlling genetic and environmental factors, it is possible to use a small sample size to establish associations between physiological or pathological conditions and corresponding changes in urine..$^{14}$ Therefore, screening potential disease biomarkers in animal models followed by validation in human samples may be a good strategy for discovering urinary biomarkers.

Aspirin remains the gold standard therapy in the prevention of myocardial infarction, ischemic stroke and vascular death among patients at both high and low risk of these events. ${ }^{8}$ Furthermore, emerging data convincingly show that in addition to its effects on cardiovascular risk, aspirin reduces cancer risk..$^{14,15}$ Due to these benefits, low-dose aspirin is widely used, and accordingly, a better understanding of its effects on the urinary proteome is necessary for advancing urinary biomarker research. Despite its benefits, a number of patients show an inadequate inhibition of platelet aggregation by aspirin, which is called aspirin resistance syndrome,${ }^{16}$ and aspirin can increase major gastrointestinal or other major extra cranial bleeding. ${ }^{17}$ Therefore, it is important to monitor platelet aggregation in patients at risk for these side effects. As urinary proteins can reflect changes in the blood coagulation status, ${ }^{4}$ we hypothesize that urinary proteomics may enable the monitoring of platelet aggregation.

In this study, we examined the effects of low-dose aspirin on the urinary proteome using label-free quantitative proteomics. Rat urine samples were collected before and at one week after aspirin administration. Urinary proteins were separated by 1D-SDS PAGE, digested in-gel based on molecular weight and identified using Nano LC-MS/MS 


\section{Materials and methods}

\section{Animals and ethics statement}

Pathogen-free, male Sprague-Dawley (180-200g) rats were purchased from the Institute of Laboratory Animal Science, Chinese Academy of Medical Science \& Peking Union Medical College (Beijing, China). All animals were fed standard laboratory diet and kept under controlled temperature $\left(22 \pm 1^{\circ} \mathrm{C}\right)$ and humidity $(65-70 \%)$. The study was performed after one week of acclimation. This study was approved by the Institute of Basic Medical Sciences Animal Ethics Committee, Peking Union Medical College (Animal Welfare Assurance Number: A5518). All rats received humane care in compliance with the institutional animal care guidelines approved by the Institutional Animal Care and Use Committee of the Peking Union Medical College.

\section{Intragastric aspirin administration and urine collection}

Urine samples from 16 rats were collected after each rat was given $1 \mathrm{~mL}$ saline by intragastric administration for $24 \mathrm{~h}$ using metabolic cages, and these samples were used as controls. The rats were then randomly divided into two groups of eight. The first group of rats received $50 \mathrm{mg} /(\mathrm{kg} / \mathrm{d})$ aspirin (low-dose (LD) group), and the second group received $200 \mathrm{mg} /(\mathrm{kg} / \mathrm{d}$ ) aspirin (high-dose (HD) group). The dosing volumes of diuretics were adjusted to $1 \mathrm{~mL}$. All rats were given aspirin by intragastric administration for one week, and urine samples were collected 7 days after aspirin administration. The samples were acidified immediately with hydrochloric acid and then cooled to $4^{\circ} \mathrm{C}$ to prevent bacterial growth and proteolysis. No rats died during the experiments. In the discovery stage, 3 samples were randomly selected and identified by LC-MS/MS due to the limited throughput of the MS, as a result, during the validation stage, we used one of these 3 samples (randomly selected) and the rest 5 samples.

\section{Platelet aggregation assay}

Blood samples were drawn from the orbital sinus and sent to the Clinical Laboratory at the Beijing Union Medical College Hospital.

\section{Acetone precipitation}

All urine samples were centrifuged at $5000 \mathrm{~g}$ for $30 \mathrm{~min}$, and the pellets were discarded. The supernatants were precipitated with $75 \% \mathrm{v} / \mathrm{v}$ acetone for $12 \mathrm{~h}$ followed by centrifugation at $12000 \mathrm{~g}$ for $30 \mathrm{~min}$. After removing the supernatant, the pellets were thoroughly air-dried, resuspended in lysis buffer $(8 \mathrm{M}$ urea, $2 \mathrm{M}$ thiourea, $50 \mathrm{mM}$ Tris and $25 \mathrm{mM}$ DTT) and subjected to protein quantitation by the Bradford assay.

\section{D-SDS PAGE and in-gel digestion}

Urinary proteins were dissolved in PAGE sample buffer $(50 \mathrm{mM}$ Tris-HCl, pH 6.8, containing 50mM DTT, $0.5 \%$ SDS, and $10 \%$ glycerol), incubated at $57^{\circ} \mathrm{C}$ for $5 \mathrm{~min}$ and resolved by $1 \mathrm{D}-\mathrm{SDS}$ PAGE. After electrophoresis, each gel band was cut into slices according to the molecular weight. The gel slices were washed twice by incubation in $100 \mathrm{mM}$ ammonium bicarbonate for $15 \mathrm{~min}$ at $37^{\circ} \mathrm{C}$, followed by $100 \mathrm{mM}$ ammonium bicarbonate/acetonitrile $(1: 1)$ for $15 \mathrm{~min}$ at $37^{\circ} \mathrm{C}$. After cooling to room temperature, the samples were reduced using $20 \mathrm{mM}$ dithiothreitol with heating at $56^{\circ} \mathrm{C}$ for 1 hour, followed by alkylation using $55 \mathrm{mM}$ iodoacetamide at room temperature for 1 hour in the dark. The gel slices were dried, and sequence-grade modified trypsin was added at a protein-to-enzyme ratio of $30: 1$, followed by incubation overnight at $37^{\circ} \mathrm{C}$. After trypsin digestion, the supernatant was collected. The resulting peptides were extracted from the gel slices by incubating once in $50 \mathrm{mM}$ ammonium bicarbonate for 1 hour at $37^{\circ} \mathrm{C}$ and twice in $5 \%$ formic acid/acetonitrile $(1: 1)$ for 1 hour at $37^{\circ} \mathrm{C}$. The three collected extractions were pooled with the initial digestion supernatant, desalted by solid-phase extraction (Oasis column; Waters, Inc., Milford, Massachusetts, USA), dried in a Speed Vac, resuspended with $10 \mu \mathrm{L} 0.1 \%$ formic acid, and stored at $-80^{\circ} \mathrm{C}$ until use.

\section{Nano LC-MS/MS analysis}

The resulting peptides were analysed by Nano LC-MS/MS using an Agilent 1200 HPLC system coupled to an LTQ-Orbitrap Velos mass spectrometer (Thermo Fisher Scientific, Bremen, and Germany). Each sample was loaded, with a maximal volume of $8 \mu \mathrm{L}$, onto a Michrom Peptide Captrap column (MW 0.5-50kD, $0.5 \times 2 \mathrm{mM}$; Michrom Bioresources, Billeria, Massachusetts, USA) in $0.1 \%$ formic acid $99.9 \%$ water at a flow rate of $20 \mu \mathrm{L} / \mathrm{min}$. The trap column effluent was transferred to a reverse-phase microcapillary column $(0.1 \times 150 \mathrm{~mm}$, packed with Magic C18, $3 \mu \mathrm{m}, 200 \AA$; Michrom Bioresources) in an Agilent 1200 HPLC system. The elution gradient for the reverse column changed from $95 \%$ mobile phase A $(0.1 \%$ formic acid, $99.9 \%$ water) to $40 \%$ mobile phase B $(0.1 \%$ formic acid, $99.9 \%$ acetonitrile $)$ within $120 \mathrm{~min}$ at a flow rate of $500 \mathrm{~nL} / \mathrm{min}$. The LTQ-Orbitrap Velos mass spectrometer was operated in data-dependent acquisition mode. Survey scan MS data were acquired using the Orbitrap in the 300-2000 $\mathrm{m} / \mathrm{z}$ range, with the resolution set to a value of 60,000 . The 20 most intense ions per survey scan were selected for CID fragmentation, and the resulting fragments were analysed in the LTQ. Dynamic exclusion was employed using a 60 second window to prevent repetitive selection of the same peptide.

\section{Database searching and protein quantification}

Mascot Daemon software (version 2.4.0, Matrix Science, London, UK) was used to search the MS/MS data against the Swiss-Prot rat database (release 2014_07; taxonomy: Rattus; containing 9354 sequences). Carbamidomethylation of cysteines was set as a fixed modification, and oxidation of methionine and protein $\mathrm{N}$-terminal acetylation were set as variable modifications. The specificity of trypsin digestion was set for cleavage after $\mathrm{K}$ or R, and two missed trypsin cleavage sites were allowed. The mass tolerances in MS and MS/MS were both set to $10 \mathrm{ppm}$ and $0.1 \mathrm{Da}$, respectively. Peptide identifications were accepted if they were detected with $90.0 \%$ probability and an FDR less than $0.1 \%$ by the Scaffold local FDR algorithm. Protein identifications were accepted if they were detected with an FDR of less than $1 \%$ and contained at least 2 identified peptides. Label-free quantification was performed using the Scaffold software (version Scaffold 4.4.0, Proteome Software Inc., Portland, OR, USA).

\section{Western blot analysis}

Two proteins that were significantly altered in urine were further verified by western blotting. A total of $30 \mathrm{mg}$ urine protein from individual samples was separated on a $10 \%$ SDS-PAGE gel and electro transferred to PVDF membranes. The coomassie staining was used as a loading control. The membranes were blocked with $5 \%$ non-fat milk and incubated overnight at $4^{\circ} \mathrm{C}$ with primary antibodies [anti-CD10 antibody (ab126593) and anti-EGF antibody (ab77851), Abcam] followed by a horseradish peroxidase-conjugated secondary antibody (155000, 074-1506, Kierkegaard and Perry Laboratories, Gaithersburg, MD, USA). Target bands were visualized using 
enhanced chemiluminescence (ECL) reagents. The ECL results were scanned and analysed using an Image Quant 400TM Imager (GE Healthcare Life Sciences, Piscataway, NJ, USA), and the intensity of each protein band was quantified using Image $J$ analysis software (National Institutes of Health, Bethesda, MD, USA).

\section{Results and discussion}

\section{Platelet aggregation assay}

Rat blood samples were collected from the orbital sinus and subjected to platelet aggregation analysis. As shown in Figure 1, the maximum platelet aggregation rates were significantly decreased after aspirin administration in both (LD and HD) groups.

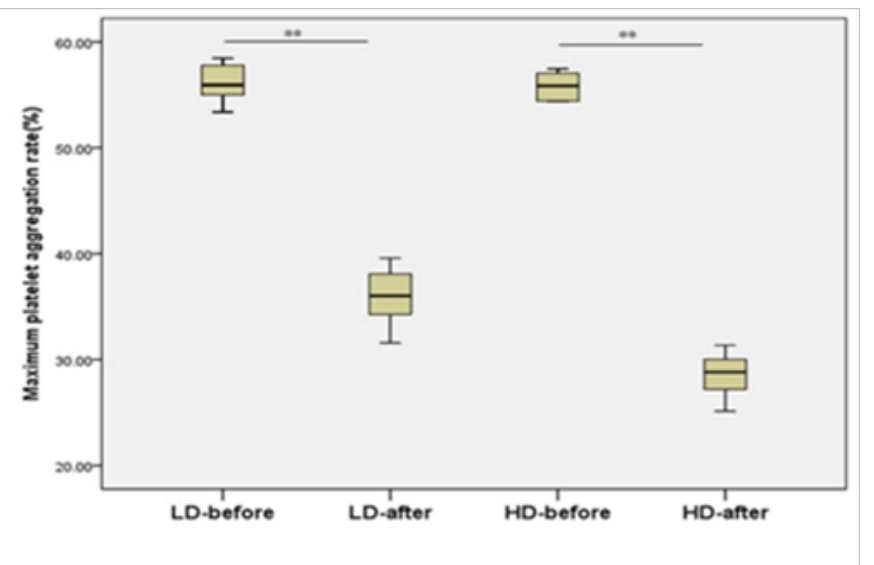

Figure I Maximum platelet aggregation rates of the low-dose and high-dose groups $(\mathrm{n}=8 ; * * \mathrm{p}<0.001)$.

\section{Urinary proteome changes in the LD aspirin group}

Urinary samples from 3 rats in the LD aspirin group were separated by 1D-SDS-PAGE, digested in-gel based on molecular weight, and identified using Nano LC-MS/MS. Each lane was cut into four slices; the slice containing proteins ranging from $14 \mathrm{kD}$ to $25 \mathrm{kD}$ contained an excess of highly abundant urinary proteins and was thus eliminated from the analysis. A total of $18 \mathrm{LC}-\mathrm{MS} / \mathrm{MS}$ runs of urine samples were performed using an LTQ-Orbitrap Velos. Due to the mass spectrometry machine time, urine samples from HD groups were only used for validation. The false discovery rate (FDR) was adjusted to be less than $1 \%$. A total of 191 proteins were identified, and the results are listed in (Table S1).

Label-free quantification was performed using the Scaffold software based on spectral counts. Based on the criteria of $p<0.05$, fold change $>2$ and spectral count $>5,12$ proteins were found to be significantly altered after aspirin administration (Table 1), suggesting that low-dose aspirin can affect the urinary proteome. Nine of the 12 $(75 \%)$ proteins have been reported as candidate disease biomarkers in previous studies; ${ }^{8}$ therefore, such effects should be taken into account in future urinary biomarker research using human urine samples. The effects of other anticoagulants, including heparin and argatroban, have been studied previously. However, protein changes induced by these anticoagulants do not overlap with those identified in the present study, suggesting that the urinary proteome can distinguish the changes in blood coagulation status caused by different coagulation mechanisms. Thus, we propose that the urinary proteome is a superior measure of blood coagulation status compared with traditional PT (prothrombin time), APTT (apart prothrombin) and TT (thrombin time) and may help in the design of more specific treatment plans for patients.

Table I Proteins significantly changed after administration of low-dose aspirin. Proteins marked with an asterisk have been previously reported as candidate disease biomarkers

\begin{tabular}{|c|c|c|c|c|c|c|c|c|}
\hline ID & Name & T-Test & $\begin{array}{l}\text { Ratl- } \\
\text { ASA }\end{array}$ & $\begin{array}{l}\text { Rat2- } \\
\text { ASA }\end{array}$ & $\begin{array}{l}\text { Rat3- } \\
\text { ASA }\end{array}$ & $\begin{array}{l}\text { Ratl- } \\
\text { CON }\end{array}$ & $\begin{array}{l}\text { Rat2- } \\
\text { CON }\end{array}$ & $\begin{array}{l}\text { Rat3- } \\
\text { CON }\end{array}$ \\
\hline \multicolumn{9}{|l|}{$>70 \mathrm{kD}$} \\
\hline P07522* & Pro-epidermal growth factor & 0.003 & 299 & 338 & 277 & 186 & 165 & 150 \\
\hline P0786 I* & CDIO & 0.026 & 22 & 25 & 29 & 14 & 11 & 10 \\
\hline PI5083 & Polymeric immunoglobulin receptor & 0.023 & 43 & 43 & 44 & 21 & 19 & 35 \\
\hline Q64230* & Meprin A subunit alpha & 0.029 & 58 & 53 & 65 & 49 & 46 & 45 \\
\hline P27590* & Uromodulin & 0.042 & 93 & 96 & 56 & 124 & 121 & 161 \\
\hline Q6P9V9 & Tubulin alpha-IB chain & 0.012 & 2 & 3 & 0 & 9 & 7 & 6 \\
\hline P607II* & Actin, cytoplasmic I & 0.016 & 27 & 34 & 26 & 54 & 44 & 64 \\
\hline P04406* & $\begin{array}{l}\text { Glyceraldehyde- } 3 \text {-phosphate } \\
\text { dehydrogenase }\end{array}$ & 0.033 & 9 & 9 & 6 & 18 & 12 & 21 \\
\hline \multicolumn{9}{|l|}{$35-70 \mathrm{kD}$} \\
\hline PI4740* & Dipeptidyl peptidase 4 & 0.016 & 3 & 2 & 2 & 5 & 5 & 4 \\
\hline P36953 & Afamin & 0.017 & 2 & 0 & 0 & 5 & 3 & 5 \\
\hline Q6IRK9 & Carboxypeptidase Q & 0.047 & 25 & 33 & 23 & 18 & 13 & 20 \\
\hline \multicolumn{9}{|l|}{$0-15 \mathrm{kD}$} \\
\hline P07I5I* & Beta-2-microglobulin & 0.023 & 6 & 6 & 10 & I & 2 & 2 \\
\hline P07522* & Pro-epidermal growth factor & 0.026 & 8 & 3 & 2 & 37 & 19 & 10 \\
\hline
\end{tabular}

Supporting information: Table SI Total proteins identified in this study. 
It is difficult to distinguish an intact protein from degraded fragments using a traditional top-down proteomic strategy, and proteins with different shear states may serve different functions. To circumvent this issue, 1D-SDS-PAGE and an in-gel digestion-based proteomic protocol were employed in the current study. According to Table 1, intact pro-EGF (pro-epidermal growth factor, molecular weight: $166 \mathrm{kD}$ ) was significantly increased after administration of aspirin; however, a fragment of pro-EGF (approximately 10kD) was decreased, which suggests that aspirin may inhibit the degradation of pro-EGF.

\section{Western blot analysis}

Pro-EGF and CD10 were selected for further validation in the other 6 rat by western blotting. Pro-EGF is associated to platelets and it was detected in platelet-rich plasma and can be secreted to the urine..$^{18}$ In previous studies, there is no correlation reported between CD10 and aspirin, however, it was changed significantly in our experiment and the mechanisms need to be further studied. As shown in Figure 2, pro-EGF $(166 \mathrm{kD})$ and CD10 were both significantly increased after aspirin administration; therefore, we propose that these two proteins may be useful in monitoring platelet aggregation. Regardless, no obvious dose-dependent relationships between the abundance of these two proteins and the dose of aspirin were observed, suggesting that other proteins affected by low-dose aspirin administration may serve as more reliable biomarkers for assessing the blood coagulation status.
A

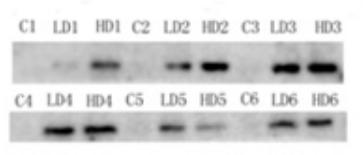

B

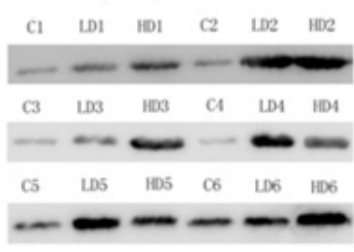

Figure 2 Western blot analysis of pro-EGF and CDIO.

$$
\text { A, pro-EGF; B, CDIO }(n=6) \text {. }
$$

\section{Conclusion}

In this study, we analysed the effects of low-dose aspirin on the urinary proteome using label-free quantitative proteomics. A total of 12 proteins were found to be significantly altered, 9 of which have been previously reported as candidate disease biomarkers. These results suggest that aspirin affects the urinary proteome and these effects must be considered in future urinary biomarker research. Protein changes induced by low-dose aspirin were distinct from those caused by heparin and argatroban treatments, suggesting that the urinary proteome can distinguish the blood coagulation status induced by different coagulation mechanisms, which may help in designing more specific treatment plans for patients. Two significantly up-regulated proteins, pro-EGF and CD10 were validated, and these proteins may be used for monitoring platelet aggregation.

\section{Acknowledgements}

This work was supported by the National Basic Research Program of China (2013CB530805; 2012CB517606; 2014CBA02005 and 2013FY114100), funds from BNU (11100704, 10300-310421102).

\section{Conflict of interest}

The author declares no conflict of interest.

\section{References}

1. Gao Y. Opinion: Roadmap to the Urine Biomarker Era. MOJ Proteomics Bioinform. 2014;1(1):00005.

2. Gao Y. Urien-an untapped goldmine for biomarker discovery? Sci China Life Sci. 2013;56(12):1145-1146.

3. Gao Y. Differences in Blood and Urine Biomarker Discovery. MOJ Proteomics Bioinform. 2015;2(5):00058.

4. Li M, Zho M, Gao Y. Changes of proteins induced by anticoagulants can be more sensitively detected in urine than in plasma. Sci China Life Sci. 2014;57(7):649-656.

5. Huang JT, Chaudhuri R, Albarbarawi O, et al. Clinical validity of plasma and urinary desmosine as biomarkers for chronic obstructive pulmonary disease. Thorax. 2012;67(6):502-508.

6. Wu T, Du Y, Han J, et al. Urinary angiostatin-a novel putative marker of renal pathology chronicity in lupus nephritis. Mol Cell Proteomics. 2013;12(5:1170-1179.

7. Gao Y. Opportunities you do not want to miss and risks you cannot afford to take in urine biomarker era. MOJ Proteomics Bioinformatics. 2014;1(1):00003

8. Shao C, Li M, Li X, et al. A tool for biomarker discovery in the urinary proteome: a manually curated human and animal urine protein biomarker database. Mol Cell Proteomics. 2011;10(11):M111 010975.

9. Guo Z, Zhang Y, Zou L, et al. A Proteomic Analysis of Individual and Gender Variations in Normal Human Urine and Cerebrospinal Fluid Using iTRAQ Quantification. PLoS One. 2015;10(7):e0133270.

10. Zhao M, Li X, Li M, et al. Effects of anesthetics pentobarbital sodium and chloral hydrate on urine proteome. Peer J. 2015;3:e813.

11. Li X, Zhao M, Li M, et al. Effects of three commonly-used diuretics on the urinary proteome. Genomics Proteomics Bioinformatics. 2014;12(3):120-126.

12. Kohler M, Franz S, Regeniter A, et al. Comparison of the urinary protein patterns of athletes by $2 \mathrm{D}-$ gel electrophoresis and mass spectrometry-a pilot study. Drug Test Anal. 2009;1(8):382-386.

13. Airoldi L, Magagnotti C, Iannuzzi AR, et al. Effects of cigarette smoking on the human urinary proteome. Biochem Biophys Res Commun. 2009;381:397-402.

14. Gao Y. Roadmap to the Urine Biomarker Era. MOJ Proteomics Bioinform. 2014;1(1):00005.

15. Ciccolini F, Svendsen CN. Fibroblast growth factor 2 (FGF-2) promotes acquisition of epidermal growth factor (EGF) responsiveness in mouse striatal precursor cells: identification of neural precursors responding to both EGF and FGF-2. J Neurosci. 1998;18(19):7869-7880.

16. Glanville RW, Qian RQ, McClure DW, et al. Calcium binding, hydroxylation, and glycosylation of the precursor epidermal growth factor-like domains of fibrillin-1, the Marfan gene protein. J Biol Chem. 1994;269(43):26630-26634.

17. Wang MH, Gonias SL, Skeel A, et al. Proteolytic activation of singlechain precursor macrophage-stimulating protein by nerve growth factorgamma and epidermal growth factor-binding protein, members of the kallikrein family. J Biol Chem. 1994;269(19):13806-13810.

18. Valcarce C, Bjork I, Stenflo J. The epidermal growth factor precursor. A calcium-binding, beta-hydroxyasparagine containing modular protein present on the surface of platelets. Eur J Biochem. 1999;260(1):200-207. 